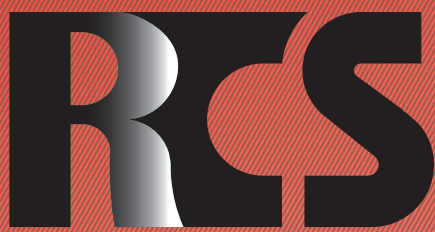

Depósito legal ppi $201502 Z U 4662$

Esta publicación científica en formato digital es continuidad de la revista impresa Depósito Legal: pp $197402 Z$ Z789

- ISSN: 1315-9518 • ISSN-E: 2477-9431

Revista de Ciencias Sociales

Universidad del Zulia. Revista de la Facultad de Ciencias Económicas y Sociales Vol. XXVII,

No. 4,2021

Esta publicación científica en formato digital es continuidad de la revista impresa Depósito Legal: pp $197402 Z 1789$ ISSN: $1315-9518$ 


\title{
Innovación en economías latinoamericanas: Análisis comparativo con respecto a Corea del Sur
}

\author{
García Ochoa, Juan José* \\ Ochoa Vazquez, Ivan ${ }^{* * *}$ \\ Valenzuela Valenzuela, Alejandro***
}

\section{Resumen}

Un gran número de investigaciones demuestran que la innovación impacta directamente en el desarrollo económico de los países. Por ello, el presente artículo tiene como objetivo analizar la brecha innovadora entre algunas naciones latinoamericanas como Brasil, Chile, Colombia, México y Perú, con referencia a Corea del Sur, identificando las variables más importantes del pilar 12 de innovación del modelo del Foro Económico Mundial. La metodología del estudio, basada en el análisis factorial multivariado, se alimenta de la información generada para las siete variables del pilar 12, en el periodo 2011-2017. Como resultado principal, se encuentra que las variables más destacadas son: La inversión de las empresas en investigación y desarrollo, la calidad de las instituciones en investigación, las patentes aplicadas, la capacidad de innovar, la colaboración universidad-industria y la disponibilidad de científicos e ingenieros. Se concluye, que Brasil, México y, en especial, Chile, han logrado desarrollar algunas capacidades innovadoras, sobre todo en materia de participación de las empresas en investigación y desarrollo, en la calidad de las instituciones de investigación y en patentes aplicadas; pero han descuidado las gestiones del gobierno para sus sectores productivos, requisito fundamental para cristalizar su posición en el ranking de innovación.

Palabras clave: Innovación; competitividad; Foro Económico Mundial; Alianza del Pacífico; análisis factorial.

\footnotetext{
* Doctor en Planeación Estratégica. Profesor Titular del Departamento de Física, Matemáticas e Ingeniería en la Universidad de Sonora, México. E-mail: juanjose.garcia@unison.mx (iD) ORCID: https://orcid.org/0000-0002-0413-7341

** Doctor en Planeación Estratégica. Profesor Asociado del Departamento de Física, Matemáticas e Ingeniería en la Universidad de Sonora, México. E-mail: ivan.ochoa@unison.mx (D) ORCID: https:// orcid.org/0000-0001-5758-6717

*** Doctor en Ciencias Sociales. Profesor Titular del Departamento de Ingeniería Industrial en la Universidad de Sonora, México. E-mail: alexval@unison.com (iD) ORCID: https://orcid.org/0000$\underline{0001-7672-9840}$
}

Recibido: 2021-06-11 · Aceptado: 2021-08-29 


\title{
Innovation in Latin American Economies: Comparative Analysis with respect to South Korea
}

\begin{abstract}
A large number of research shows that innovation directly impacts the economic development of countries. This article aims to analyze the innovation gap between some Latin American nations such as Brazil, Chile, Colombia, Mexico and Peru, with reference to South Korea identifying the most important variables of pillar 12 of innovation of the World Economic Forum model. The study methodology, based on multivariate factor analysis, is fed with information generated for the seven variables of pillar 12 , in the period 2011-2017. As the main result, it is found that the most prominent variables are: Investment by companies in research and development, the quality of research institutions, applied patents, the ability to innovate, university-industry collaboration and the availability of scientists and engineers. It is concluded that Brazil, Mexico and, especially, Chile, have managed to develop some innovative capacities, especially in terms of the participation of companies in research and development, in the quality of research institutions and in applied patents; but they have neglected the government's efforts for their productive sectors, a fundamental requirement to crystallize their position in the innovation ranking.
\end{abstract}

Keywords: Innovation; competitiveness; World Economic Forum; Pacific alliance; factorial analysis.

\section{Introducción}

Aun cuando la innovación está ligada al desarrollo humano, solo hace unas pocas décadas logra relevancia como categoría de análisis de la realidad social y, en particular, del desarrollo económico. En ese campo, Schumpeter (1967) se destaca como pionero puesto que, desde su Teoría del desenvolvimiento económico, identificó a la innovación (tanto tecnológica como social) como uno de los principales motores del desarrollo económico. Como era lógico, el análisis de la innovación ha bajado del nivel general o macro de las economías hacia el nivel microeconómico en las empresas (Montoya, 2004; Vega et al., 2005; Parada, Ganga y Rivera, 2017; Navarro-Caballero et al., 2020), creándose un sistema interactivo empresasentorno que ha llevado a la concepción de los sistemas de innovación (Nelson y Winter, 1982; Freeman, 1987; Bengt-Åke, 1992).
Sin embargo, hasta hoy en día, no hay una definición única entre los especialistas, aun cuando ha habido un esfuerzo considerable para homogenizar criterios (Organización para la Cooperación y el Desarrollo Económicos [OCDE], 2005), creándose un consenso acerca de que la innovación se asocia con la introducción de un producto o servicio nuevo o mejorado, un proceso nuevo, una práctica de mercado, o una forma de organización (Pino et al., 2016; Geldes, Felzensztein y Palacios-Fenech, 2017). Así mismo, aunque se han realizado esfuerzos considerables con ese propósito, no existe consenso sobre los métodos para evaluar empíricamente la innovación, ni a nivel de las empresas, ni al de economías o países.

Uno de esos esfuerzos es la construcción del Índice Global de Competitividad (IGC), que el Foro Económico Mundial publica cada año. Este estudio se basa en la edición del año 2017, la cual provee un marco metodológico 
para el estudio de la competitividad que permite el ordenamiento (o ranking) con base en 112 indicadores, agrupados en 25 factores que constituyen, a su vez, 12 pilares que muestran los fundamentos microeconómicos y macroeconómicos de la competitividad de la nación. De esos pilares, el número 12 es un índice construido con siete variables, que se describen en los siguientes apartados, y enfoca, específicamente, en la innovación.

Esos índices globales han sido utilizados por académicos, empresarios y gobiernos en la fundamentación de investigaciones y el diseño de políticas públicas con el fin de implementar estrategias de desarrollo regional (CastroGonzález, Peña-Vinces y Guillen, 2016; Jiménez y Geldes, 2019). Sin embargo, han sido fuertemente criticados por autoridades académicas sobre el tema, como Lall (2001); $y$, Peña-Vinces (2009), quienes enfocan esas críticas, principalmente, en la debilidad del análisis estadístico sobre el que se construyen y, específicamente, en la asignación arbitraria de los ponderadores para la construcción del indicador.

Asimismo, el interés de las naciones en la innovación ha sido desigual, presentando fuertes retrasos en áreas muy extensas del mundo, como América Latina. En esta región, los primeros estudios se iniciaron por el año 2000 (Oliver y Stezano, 2017; Heredia et al. 2018), no obstante, aunque el interés por el tema ha crecido desde entonces, las publicaciones científicas enfocadas en ese campo siguen siendo escasas.

Por tal razón, el objetivo principal del presente trabajo es analizar la brechainnovadora entre algunas naciones latinoamericanas como Brasil, Chile, Colombia, México y Perú, con referencia a Corea del Sur, identificando las variables más importantes del pilar 12 de innovación del modelo del Foro Económico Mundial (WEF).

\section{Innovación y competitividad de los países}

El concepto de innovación ha adquirido fuerza y relevancia en las aportaciones teóricas de la comunidad científica interesada en el desarrollo económico y social de los países (Parada et al., 2017), a partir de los estudios pioneros de Schumpeter (1967), quien fue el economista austriaco que le asignó a la "destrucción creativa" el papel fuerza causante del desarrollo (Montoya, 2004).

Al respecto, un resultado crucial de la innovación es la competitividad entre las naciones, tópico cuya relevancia conceptual se incrementa a la par del desarrollo del comercio internacional. Con respecto a estudios de competitividad, el pionero fue Porter (1990), quien desarrolló las bases teóricas de la misma en su famoso libro sobre: "La Ventaja Competitiva de la Naciones", e hizo depender la competitividad de la productividad de bienes y servicios y, de ambos, la prosperidad en una nación. En otro importante documento, Porter (2008) también destaca el fundamento microeconómico de la competitividad (cambio tecnológico en las empresas, innovación, elevación de la productividad del trabajo) para la política económica nacional. En este argumento, la competitividad depende de la productividad, la cual a su vez se basa en dos pilares: La innovación y la calidad del ambiente microeconómico.

Sin embargo, siendo la innovación la causa última de la productividad y la competitividad, su instrumentación no es fácil ni a nivel de países ni de empresas. El mismo Foro Económico Mundial, reconoce que el pilar de la innovación es el que menor desempeño registra (su puntaje es menor que el de los 11 pilares restantes). La razón de ese relativamente pobre desempeño, argumenta el organismo, es que la innovación es un proceso complejo que pasa por diferentes etapas: La generación de ideas, la invención, la inversión y el mercado. Dentro de un ecosistema de innovación, la realización de las etapas depende de diferentes factores, si alguno de los factores falla, frena el paso de las ideas a las etapas sucesivas (WEF, 2017).

En la Tabla 1, se presentan los puntajes que reporta el Foro Económico Mundial de cada uno de los países seleccionados (Brasil, Chile, 
Colombia, México, Perú y Corea del Sur), en las siete variables que constituyen este pilar número 12 de innovación durante el periodo 2011-2017. Las siete variables que constituyen el índice del pilar 12 son las siguientes: $\mathrm{X}_{1}$, capacidad de innovar; $\mathrm{X}_{2}$, calidad de las instituciones de investigación; $\mathrm{X}_{3}$, inversión de las empresas en investigación y desarrollo; $\mathrm{X}_{4}$, colaboración universidad-industria; $\mathrm{X}_{5}$, gestión del gobierno en tecnología avanzada; $\mathrm{X}_{6}$, disponibilidad de científicos e ingenieros, $\mathrm{y} \mathrm{X}_{7}$, patentes aplicadas.

Tabla 1

Variables del pilar 12 de Innovación del WEF

\begin{tabular}{|c|c|c|c|c|c|c|c|c|}
\hline País & Año & $\mathbf{X 1}$ & $\mathrm{X} 2$ & $\mathrm{X} 3$ & $\mathrm{X} 4$ & X5 & X6 & $\mathbf{X} 7$ \\
\hline Brasil & 2011 & 3,8 & 4,1 & 3,8 & 4,2 & 3,9 & 3,8 & 0,9 \\
\hline Chile & 2011 & 3 & 4 & 3,1 & 4,1 & 4 & 4,7 & 1,3 \\
\hline Colombia & 2011 & 3,2 & 3,6 & 3 & 4,1 & 4 & 4 & 0,1 \\
\hline Corea del Sur & 2011 & 4,3 & 4,8 & 4,8 & 4,7 & 4,1 & 4,9 & 241 \\
\hline México & 2011 & 3 & 4 & 3 & 4 & 3,5 & 3,9 & 0,9 \\
\hline Perú & 2011 & 2,7 & 2,9 & 2,6 & 3,2 & 3,3 & 3,5 & 0 \\
\hline Brasil & 2012 & 3,7 & 4,1 & 3,6 & 4,1 & 3,8 & 3,5 & 2,8 \\
\hline Chile & 2012 & 3 & 4,2 & 3,2 & 4,2 & 3,9 & 4,7 & 3,8 \\
\hline Colombia & 2012 & 3,2 & 3,4 & 3,1 & 4 & 3,8 & 3,7 & 1,1 \\
\hline Corea del Sur & 2012 & 4,5 & 4,9 & 4,9 & 4,7 & 4 & 4,9 & 161 \\
\hline México & 2012 & 3,1 & 4 & 3,2 & 4,1 & 3,6 & 4 & 1,6 \\
\hline Perú & 2012 & 2,8 & 2,8 & 2,6 & 3,1 & 3,2 & 3,4 & 0,2 \\
\hline Brasil & 2013 & 4 & 4,3 & 3,6 & 4 & 3,5 & 3,4 & 2,9 \\
\hline Chile & 2013 & 3,5 & 4,1 & 3,2 & 4,3 & 4 & 4,7 & 5,7 \\
\hline Colombia & 2013 & 3,4 & 3,3 & 3,1 & 3,8 & 3,7 & 3,7 & 1,1 \\
\hline Corea del Sur & 2013 & 4,5 & 4,9 & 4,6 & 4,7 & 4 & 4,6 & 183 \\
\hline México & 2013 & 3,5 & 4 & 3,2 & 4,1 & 3,6 & 4 & 1,7 \\
\hline Perú & 2013 & 3,1 & 2,9 & 2,5 & 3,1 & 3,2 & 3,4 & 0,2 \\
\hline Brasil & 2014 & 4,1 & 4 & 3,5 & 3,3 & 3,4 & 3,3 & 3,2 \\
\hline Chile & 2014 & 3,7 & 4 & 3,1 & 4,2 & 3,8 & 4,6 & 6,7 \\
\hline Colombia & 2014 & 3,5 & 3,5 & 3 & 3,9 & 3,7 & 3,8 & 1,2 \\
\hline Corea del Sur & 2014 & 4,7 & 5 & 4,5 & 4,6 & 4,1 & 4,4 & 202 \\
\hline México & 2014 & 3,7 & 3,9 & 3,1 & 4 & 3,4 & 3,9 & 1,8 \\
\hline Perú & 2014 & 3,4 & 2,9 & 2,6 & 3,1 & 3 & 3,3 & 0,3 \\
\hline Brasil & 2015 & 3,8 & 3,6 & 3,3 & 3,8 & 3,1 & 3,3 & 3,5 \\
\hline Chile & 2015 & 3,8 & 4,1 & 3 & 4,2 & 3,1 & 4,6 & 7,1 \\
\hline Colombia & 2015 & 3,7 & 3,7 & 2,9 & 3,9 & 3,3 & 3,8 & 1,4 \\
\hline Corea del Sur & 2015 & 4,8 & 4,8 & 4,6 & 4,6 & 3,9 & 4,4 & 221 \\
\hline México & 2015 & 4 & 4,1 & 3,2 & 4 & 3,1 & 4,1 & 1,9 \\
\hline Perú & 2015 & 3,6 & 2,9 & 2,7 & 3,1 & 2,7 & 3,2 & 0,3 \\
\hline Brasil & 2016 & 3,9 & 3,6 & 3,2 & 3,2 & 2,7 & 3,4 & 3,5 \\
\hline Chile & 2016 & 3,9 & 4,3 & 2,9 & 3,5 & 2,7 & 4,7 & 7,5 \\
\hline Colombia & 2016 & 3,9 & 3,8 & 2,9 & 3,7 & 3,1 & 3,9 & 1,9 \\
\hline Corea del Sur & 2016 & 4,8 & 4,6 & 4,5 & 4,4 & 3,7 & 4,4 & 233 \\
\hline México & 2016 & 4,1 & 4,3 & 3,2 & 3,6 & 3 & 4,1 & 2 \\
\hline Perú & 2016 & 3,7 & 3,1 & 2,7 & 2,9 & 2,6 & 3,4 & 0,5 \\
\hline Brasil & 2017 & 4,1 & 3,7 & 3,4 & 3,4 & 2,7 & 3,6 & 3,4 \\
\hline Chile & 2017 & 4 & 4,4 & 3 & 3,5 & 2,8 & 4,8 & 8,8 \\
\hline Colombia & 2017 & 3,8 & 3,9 & 3,1 & 3,6 & 3,2 & 3,9 & 2,1 \\
\hline Corea del Sur & 2017 & 4,7 & 4,8 & 4,4 & 4,4 & 2,8 & 4,5 & 250 \\
\hline México & 2017 & 4,1 & 4,3 & 3,2 & 3,6 & 3,1 & 4,2 & 2,4 \\
\hline Perú & 2017 & 3,6 & 3,2 & 2,7 & 2,9 & 2,7 & 3,5 & 0,6 \\
\hline
\end{tabular}

Fuente: Elaboración propia, 2021 con base en datos del WEF (2011-2017). 
Sobre la base del constructo No.12 del Foro Económico Mundial, ha habido un conjunto reducido de trabajos cuyo propósito es el estudio comparado de diversas economías en función de las capacidades innovadoras. En ese sentido, Arredondo, Vázquez y De la Garza (2016), realizaron un estudio comparativo entre los países de la Alianza del Pacífico (AP) (Chile, Colombia, México y Perú) y otras 17 naciones que no pertenecen a ese organismo de coordinación, utilizando los indicadores del pilar de innovación del WEF (2014). La comparación fue realizada mediante el análisis de varianza y encontraron que las patentes (variable $\mathrm{X}_{7}$ ) es la que caracteriza o identifica a los países de la AP como más innovadores en relación con otro grupo formado por 17 países de América Latina (AL).

No obstante, en el análisis de varianza, las patentes resultaron estadísticamente no significativas en la explicación global de la innovación. Una carencia en dicho trabajo es, por una parte, que no se identifica cuáles países son los más innovadores entre los grupos $\mathrm{y}$, por otra parte, no se considera un estudio longitudinal para identificar a los más innovadores del total de países analizados.

Asimismo, tanto León y Ramírez (2014); como Cazallo y Salazar (2018), encontraron que entre los países de América Latina pertenecientes a la Alianza del Pacífico (AP), hay un intercambio comercial más intenso que en los otros países de la región con los mercados de América del norte, Europa y Asia del este/sureste. No obstante, encontraron también que las políticas económicas de los países de la AP no se centran en el impulso a cadenas de valor (sobre todo las dirigidas por empresas trasnacionales formadas con capitales de los países miembros). Así, la inmensa mayoría de las empresas se constituyen como organizaciones aisladas, con capitales locales.

Por su parte, Cazallo et al. (2019) compararon dos bloques de países dentro de la región: Los que pertenecen al Mercosur (Brasil, Argentina, Uruguay y Paraguay), y los miembros de la AP, en el periodo 2012 al 2017. El propósito era saber cuáles pilares (y sus indicadores) de los 12 que componen el reporte del WEF, son los más influyentes en el nivel de competitividad. Por medio del análisis discriminante, identificaron que tres son los pilares que más influyeron: El entorno macroeconómico (pilar 3); el de salud y educación primaria (pilar 4), y el tamaño del mercado (pilar 10). En términos comparativos, también observaron que la dispersión de los resultados es menos amplia (competitividad más consistente) para los países de la AP que en los del Mercosur.

Adicionalmente, Castro-González et al. (2016) aplican el modelo del Doble Diamante (DD) de Moon, Rugman y Verbeke (1998), que a su vez se basa en el modelo del diamante de Porter (1990), para investigar el estado de la competitividad en algunos países de América Latina (Argentina, Bolivia, Brasil, Chile, Colombia, Ecuador, Paraguay, Perú, Uruguay y Venezuela). A diferencia del Foro Económico Mundial, que ubica en primer lugar a Chile, el orden que ellos encontraron es el siguiente (de mayor a menor): Brasil, Argentina, Chile, Uruguay, Colombia, Venezuela, Ecuador, Perú, Paraguay y Bolivia.

Dichos hallazgos se basan en el promedio de la información de los años 2000 al 2010 reportada por tres fuentes: Banco Mundial (BM), Fondo Monetario Internacional (FMI) y Comisión Económica para América Latina y el Caribe (CEPAL). En este estudio, a diferencia de los hallazgos basados en los indicadores del Foro Económico Mundial, encuentran que sí resultó estadísticamente significativo para la competitividad tanto el gasto en investigación y desarrollo, así como la aplicación de patentes por residentes.

En un estudio similar, pero limitado a Chile y a Perú (Peña-Vinces, 2009), también utilizando el modelo del Doble Diamante alimentado con información de las mismas instituciones (BM, FMI y CEPAL), considerando además a la Organización de las Naciones Unidas, los autores encuentran que Perú es más competitivo que Chile en el diamante nacional; mientras que, al revés, Chile es más competitivo que Perú en el diamante internacional. 
Utilizando datos del Banco Mundial, Banco Interamericano de Desarrollo y documentos institucionales públicos, RojasSantoyo, Elizalde-Bobadilla y Jiménez-García (2018), compararon las políticas del desarrollo productivo y fomento a la innovación de Corea del Sur con Brasil y Colombia. Confirmaron que las capacidades humanas y tecnológicas eran superiores en el país asiático, debido a una mayor proporción del PIB (4,55\%) destinado a la educación. Mientras que, en Colombia, en el mismo periodo, había sido de apenas el 0,24\% y en Brasil de 1,26\%.

Por último, Oliver y Stezano (2017) realizaron una investigación bibliométrica para explorar la evolución de la innovación en el periodo 2010-2017 de Brasil, Chile y México, los tres países considerados más desarrollados en Latinoamérica. La metodología de ese estudio consistió en la búsqueda bibliográfica en la base de datos Scopus con referencia a la palabra innovación y sus variantes. En Brasil, según los resultados, se produjeron 815 documentos, 87 en Chile y 177 en México. Un resultado adicional, es que la mayoría de las publicaciones se concentraron en tres áreas: Ciencias sociales, economía (incluyendo econometría y finanzas), y negocios (incluyendo gestión y contabilidad). Este resultado es además un indicador del grado de importancia del tema de la innovación en revistas selectas de investigación científica.

\section{Metodología}

En esta sección se expone los criterios para la elección de los países que delimitan este ámbito de estudio, se explican las fuentes de información recabadas durante el periodo desde donde se extraen las variables, y se presenta el modelo estadístico utilizado.

\subsection{El marco de los países}

Como ya se mencionó anteriormente, los países de este estudio son, por un lado, Corea del Sur (que registra un desempeño ejemplar dentro de las 35 economías más innovadoras del mundo) según Nikzad (2013); y WEF (2017); y, por otro, Brasil (que forma parte de los llamados BRICS, acrónimo por Brasil, Rusia India, China y Sudáfrica), además de Chile, Colombia, México y Perú, países que constituyen la llamada Alianza del Pacífico.

Todos estos países impulsan, en mayor o menor grado, políticas públicas cuyo objetivo es el mejoramiento de la innovación y competitividad (Oropeza, 2011; Arredondo et al., 2016); por ello, y dentro del propósito de este artículo, se realizará el análisis comparado respecto al desempeño innovador de algunos de ellos respecto de Corea del Sur.

\subsection{Fuentes de información y variables}

En esta investigación se utilizan los datos generados por el Foro Económico Mundial sobre competitividad e innovación en el periodo 2010 al 2017. Dichos reportes están construidos con una gran cantidad de variables, organizadas en factores e indicadores, tanto micro como macro, que miden el desempeño de la prosperidad de 140 países, mediante los 12 pilares de competitividad ya mencionados, cuyos ámbitos del desarrollo competitivo son el de recursos básicos, la eficiencia y la innovación.

La comparación del desempeño innovador de los países analizados se hará mediante los resultados mostrados por cada uno de ellos en el pilar 12 del Índice de Competitividad del Foro Económico Mundial. Como ya se dijo, este índice (el pilar 12) está compuesto por siete variables, de la $\mathrm{X}_{1}$ a la $\mathrm{X}_{7}$, cuya denominación se proporcionó en la Tabla 1.

\subsection{Métodos de análisis}

La información se ha estudiado utilizando el análisis factorial (la reversa, por así decirlo, del modelo de regresión lineal múltiple, como parte, del análisis multivariado). A través de esa metodología, se 
calculan la asignación de ponderaciones a las variables involucradas.

La presentación técnica del modelo consiste en que cada variable observable de un vector, dígase $\mathrm{X}$, se relacionan con una única variable latente llamada $\mathrm{f}$ (por factor). Así, el análisis factorial se puede ver en forma matricial en la siguiente ecuación:

$$
\begin{aligned}
& \boldsymbol{X}=\wedge \boldsymbol{f}+\boldsymbol{u} \\
& \text { Donde: }
\end{aligned}
$$

$\mathrm{X}$ es un vector $(\mathrm{px} 1)$ de variables observables de una población de interés.

$\Lambda$ es una matriz (pxn) de coeficientes desconocidos o cargas factoriales.

$f$ es un vector $(\mathrm{nx} 1)$ de variables latentes $o$ factores no observados.

$u$ es un vector $(\mathrm{px} 1)$ de perturbaciones no observadas.

Con el propósito de asegurar la validez del estudio, la matriz de datos es sometida a pruebas de adecuación para el análisis multivariado. Esas pruebas son la de esfericidad de Bartlett para determinar si se trata de una matriz identidad, en cuyo caso no se puede utilizar el análisis factorial. Asimismo, una vez descartada la existencia de la matriz identidad, se determina el grado de correlación general entre las variables por medio de la prueba Kaiser-Meyer-Olkin (KMO). Los niveles de correlación $0.5<\mathrm{KMO}$ $\leq 1$ indican cuáles variables deberían incluirse en el análisis (Cleff, 2019).

Con base en las pruebas anteriores, se lleva a cabo el análisis de dendograma (o niveles de grupos). Esta técnica multivalente no funciona para diferenciar las variables relevantes de las irrelevantes. Su utilidad consiste en que clasifica las variables similares en grupos o categorías, de tal manera que un grupo de variables debe ser diferente de otro (Hair et al. 2019).

\section{Resultados y discusión}

En la Tabla 2, se puede verificar que existe correlación entre las variables analizadas, puesto que, en la prueba de Bartlett, el nivel crítico del valor $p$ es muy inferior a $0,01 \%$. Adicionalmente, se aplica el análisis de adecuación o suficiencia de muestreo general (KMO) y la prueba correspondiente arroja un valor de 0,716. Aquí el criterio es que cada variable es cabalmente pronosticada por las demás variables si KMO llega a 1, el valor obtenido se considera aceptable de acuerdo con Cleff (2019). Por lo tanto, este trabajo resulta consistente y adecuado dado los resultados de los análisis estadísticos generados.

Tabla 2

Prueba de Kaiser-Meyer-Olkin (KMO) y de Bartlett para los datos del período,

\begin{tabular}{|c|c|c|c|c|}
\hline \multirow[b]{2}{*}{ Determinante } & \multirow{2}{*}{$\begin{array}{c}\begin{array}{c}\text { Prueba de } \\
\text { KMO }\end{array} \\
2016 \\
\end{array}$} & \multicolumn{3}{|c|}{ Prueba de Bartlett } \\
\hline & & Chi-cuadrada & $\begin{array}{c}\text { Grados de } \\
\text { libertad }\end{array}$ & Valor $\mathrm{p}$ \\
\hline Innovación global, pilar 12 & 0,716 & 417.479 & 28 & 0.000 \\
\hline
\end{tabular}
2011-2017

Fuente: Elaboración propia, 2021.

Además, de acuerdo con los resultados mostrados en la Tabla 3, el análisis factorial mediante el método de cálculo por componentes principales, está bien representado por dos factores que explican el $84,506 \%$ de la varianza total de la información, es decir, del comportamiento de los indicadores. Lo anterior concuerda con el criterio de Kaiser para determinar el número apropiado de factores, que toman en cuenta sólo los factores con eigenvalores $\geq 1$ (Cleff, 2019). 


\section{Tabla 3}

Varianza total explicada y determinación de los factores comunes para la evaluación del pilar 12 de las entidades, durante el periodo de 2011-2017

\begin{tabular}{|c|c|c|c|c|c|c|}
\hline \multirow{2}{*}{$\begin{array}{c}\text { Componentes } \\
\text { de la } \\
\text { Innovación } \\
\text { global, pilar } \\
12\end{array}$} & \multicolumn{3}{|c|}{ Autovalores iniciales } & \multicolumn{3}{|c|}{$\begin{array}{c}\text { Suma de las saturaciones al cuadrado de } \\
\text { la rotación }\end{array}$} \\
\hline & Total & $\begin{array}{l}\% \text { de la } \\
\text { varianza }\end{array}$ & $\begin{array}{c}\% \\
\text { acumulado }\end{array}$ & Total & $\begin{array}{l}\% \text { de la } \\
\text { varianza }\end{array}$ & $\begin{array}{c}\% \\
\text { acumulado }\end{array}$ \\
\hline 1 & 4.841 & 60.509 & 60.509 & 4.654 & 58.181 & 58.181 \\
\hline 2 & 1.920 & 23.997 & 84.506 & 2.106 & 26.325 & 84.506 \\
\hline 3 & 0.680 & 8.504 & 93.010 & & & \\
\hline 4 & 0.288 & 3.600 & 96.609 & & & \\
\hline 5 & 0.154 & 1.928 & 98.537 & & & \\
\hline 6 & 0.048 & 0.602 & 99.138 & & & \\
\hline 7 & 0.045 & 0.559 & 99.697 & & & \\
\hline 8 & 0.024 & 0.303 & 100.000 & & & \\
\hline
\end{tabular}

Nota: Método de rotación por varımax con Kaiser, con SPSS, V.22.

Fuente: Elaboración propia, 2021.

Una vez comprobada la validez y confiabilidad del análisis factorial, según los resultados anteriores, para el análisis e interpretación de los resultados se consideran las comunalidades y la matriz de componentes rotada, que muestran las correlaciones entre cada variable y el factor correspondiente. Esos resultados se muestran en la Tabla 4 .

Tabla 4

Matriz de componentes reducidos y rotados

\begin{tabular}{cccccccccc}
\hline \multirow{2}{*}{ Factor } & \multicolumn{7}{c}{ Coeficientes para el cálculo del índice de innovación global, pilar 12 } \\
\cline { 2 - 10 } & $\mathbf{A}$ & $\mathbf{X}_{1}$ & $\mathbf{X}_{\mathbf{2}}$ & $\mathbf{X}_{\mathbf{3}}$ & $\mathbf{X}_{\mathbf{4}}$ & $\mathbf{X}_{\mathbf{5}}$ & $\mathbf{X}_{\mathbf{6}}$ & $\mathbf{X}_{7}$ \\
\hline $\mathrm{Z}_{1}$ & 0.127 & 0.851 & 0.931 & 0.933 & 0.805 & 0.523 & 0.704 & 0.871 \\
$\mathrm{Z}_{2}$ & -0.949 & -0.456 & 0.076 & 0.143 & 0.521 & 0.804 & 0.229 & 0.020 \\
Comunalidad & .917 & .933 & .873 & .891 & .920 & .920 & .548 & .759 \\
\hline
\end{tabular}

Nota: Método de extracción por componentes principales, con SPSS, V.22; y método de rotación por normalización varimax con Kaiser.

Fuente: Elaboración propia, 2021. 
Para interpretar apropiadamente la Tabla 4, considere que A es la variable tiempo expresada en los años del período y que las siete variables indicadoras ( $\mathrm{de}_{\mathrm{X}_{1}}$ a $\mathrm{X}_{7}$ ) son como se especifica en la Tabla 1. En particular, todas las variables con altos valores en sus coeficientes de correlación con excepción de A y $\mathrm{X}_{5}$, se agrupan de manera más estrecha al factor $Z_{1}$, que se refiere a la capacidad inventiva e innovadora del país. Mientras que A y $\mathrm{X}_{5}$ (que en forma conjunta se refieren a la gestión del gobierno en el transcurso del tiempo), se relacionan de manera más fuerte con el factor $\mathrm{Z}_{2}$. Con estos dos factores, es posible estimar el ranking de innovación global de los países (el pilar 12 del WEF).

En ese sentido, la ecuación 2 está constituida por todos los coeficientes calculados en la Tabla 4, no obstante, aquí utilizaremos el factor $\left(Z_{1}\right)$ como criterio de agrupación y clasificación de los países, puesto que es el factor asociado con la innovación.

$Z_{1}=0.127 A+0.851 X_{1}+0.931 X_{2}+0.933 X_{3}+0.805 X_{4}+0.523 X_{5}+0.704 X_{6}+0.871 X_{7}$

Los valores de los coeficientes permiten detectar que las seis variables más influyentes en la innovación, agrupados en $Z_{1}$, son las que determinan la trayectoria de los países. En este campo, ya se ha encontrado que existe una relación causal entre la innovación de los países y la variable 3 (gastos en innovación), la variable 4 (colaboración entre universidadindustria) y la variable 7 (aplicación de patentes) (Arredondo et al., 2016; CastroGonzáles et al. 2016; López, 2019).

En congruencia con lo anterior, se ha encontrado también que hay una relación entre los gastos en investigación, como porcentaje del PIB, con la publicación de patentes y artículos, por un lado, y con la exportación de productos de alta tecnología, por el otro (Olavarrieta y Villena, 2014).

Para calcular los grupos (o dendograma), primero se verifica la ecuación 2 multiplicando la matriz de datos de la Tabla 1 por el vector transpuesto de coeficientes de la ecuación mencionada. El resultado de lo anterior es el índice de innovación anual de los países el cual, mediante el método de Ward y distancia Euclidiana, identifica los grupos cuya distinción es la similitud de características dentro de cada uno de ellos.

El Gráfico I, muestra el índice de innovación construido con la ecuación 2 para cada país de la comparación. Un patrón evidente de esperarse es que Corea está por encima de todos los países seleccionados de América Latina, en fuerza innovadora, durante todos los años del periodo. Además, mantiene un nivel aproximadamente constante a lo largo de los años seleccionados, con una ligera caída que hace que el 2017 esté un poco por debajo de 2011, sin embargo, dentro de la banda de los 90 a 100 puntos. 


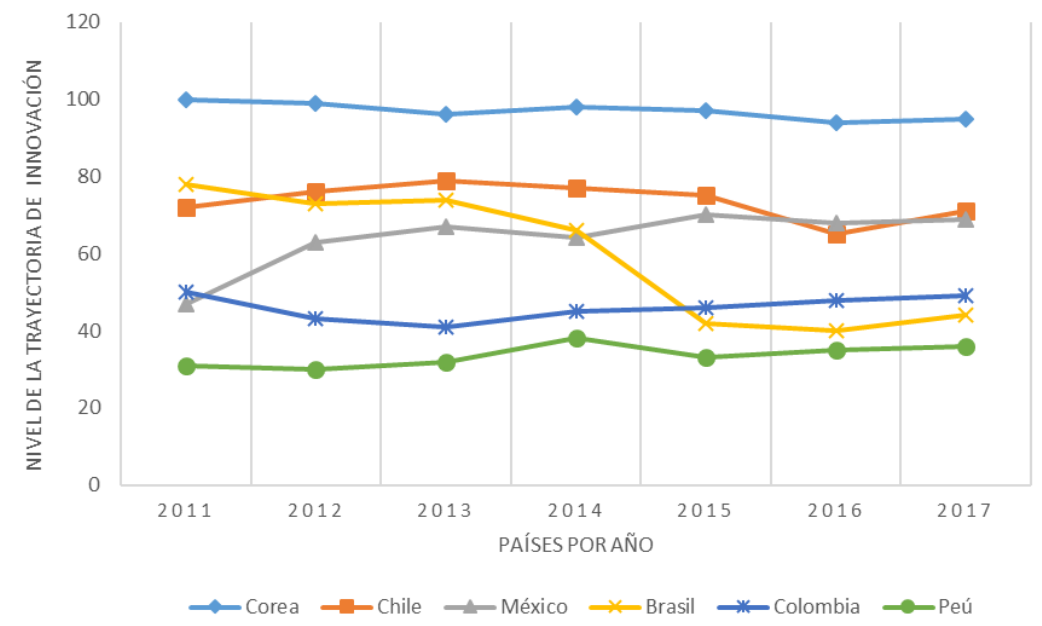

Nota: Método de clasificación: Análisis de conglomerados jerárquicos, con SPSS, V.22. Método de conglomeración: Ward; con medida de distancia euclídea

Fuente: Elaboración propia, 2021.

\section{Gráfico I: Países agrupados en cuatro niveles de su trayectoria en innovación, 2011-2017}

Los países de América Latina, por su parte, muestran desigualdades considerables $\mathrm{y}$, dentro de estos, en la sección de la banda de los 20 a los 39 puntos, se identifica a Perú, mientras que Colombia se identifica claramente en toda su trayectoria dentro de la banda de los 40 a los 59 puntos. Algunos comportamientos erráticos se visualizan para el caso de Brasil, que ha visto disminuida su posición respecto a otros países, al bajar su puntuación desde los 78 a los 44 puntos, es decir, cae de la banda identificada entre los 60 a los 80 puntos. Por el contrario, México es el único país del grupo que mejoró sustancialmente sus capacidades innovadoras a lo largo del periodo y, desde el 2012, se le identifica en la banda de los 60 a los 80 puntos; finalmente, se observa que Chile se mantiene consistentemente en su fuerza innovadora a lo largo del periodo dentro de la banda de los 60 a los 80 puntos.

\section{Conclusiones}

Con base en el presente estudio se puede afirmar que, el país de referencia, Corea del Sur ha construido sus capacidades innovadoras desde un nivel de marginalidad en el concierto de las naciones, lo que permite imaginar que países como los que se comparan (México, Brasil, Chile, Colombia y Perú), tienen una ruta para alcanzar niveles que les 
permita mejorar su competitividad innovadora $\mathrm{y}$, por tanto, su participación en el comercio internacional.

Además, la comparación es útil porque el índice de referencia (el pilar 12 del Índice Global de Competitividad del Foro Económico Mundial), es usado para el diseño de políticas públicas que buscan fomentar la innovación. Fundamentalmente, el estudio indica que un país podría avanzar en su capacidad de innovar (Variable $\mathrm{X}_{1}$ ), priorizando la educación (que se relaciona con las variables $\mathrm{X}_{2} \mathrm{y}_{6}$ ) a través de un incremento del gasto en ese rubro respecto al PIB, fomentando la innovación en las empresas (variables $\mathrm{X}_{3}, \mathrm{X}_{4}, \mathrm{X}_{5} \mathrm{y} \mathrm{X}_{7}$ ).

Los altos valores de correlación de las variables, en especial en inversión de las empresas en investigación y desarrollo; calidad de las instituciones en investigación y patentes aplicadas, son las que más han contribuido en el posicionamiento innovador de países como: Corea, Chile, Brasil y México. Lo que sugiere que un país como Perú, puede pasar al nivel de México o Chile, enfocándose en el impulso de políticas relacionadas principalmente con dichas variables.

Los resultados por países muestran que los niveles alcanzados ni son definitivos ni son fatales. Es decir, habiendo alcanzado un nivel bueno en comportamiento innovador, nada garantiza su reducción (como es el caso de Brasil) y, al revés, un nivel bajo en el índice es un estímulo para mejorar el puntaje (como es el caso de México en ese periodo del 2011 al 2017). Además, los resultados expuestos dan cuenta que los esfuerzos para incrementar el perfil innovador de los países debe ser una política de Estado, donde participen la sociedad, las empresas, la academia y la ciencia. Los casos de Colombia y Perú, son ilustrativos o de la carencia de esfuerzos efectivos o de una inercia muy fuerte refractaria a la innovación.

A pesar de los resultados disparejos en los países de América Latina, los datos muestran algunas persistencias. Además, de la mencionada respecto a Colombia y Perú, y a pesar de la reducción de Brasil, este país, junto con México y Chile, se mantienen en un nivel en promedio de desarrollo innovador.
Lo que se puede desprender de este hecho, es que los países generan una masa crítica de conocimientos, procesos e inversiones que fomentan la innovación que les permite generar una dinámica innovadora. La parte más interesante de esta conclusión es que un país, como Brasil, que baja al nivel de Colombia, está en mejores condiciones para despegar con respecto de los últimos dos países.

Adicionalmente, se puede decir que Brasil, México y, en especial, Chile, han logrado desarrollar algunas capacidades innovadoras, sobre todo en materia de participación de las empresas en investigación y desarrollo, en la calidad de las instituciones de investigación y en patentes aplicadas; pero han descuidado las gestiones del gobierno para sus sectores productivos, requisito fundamental para cristalizar su posición en el ranking de innovación.

Por último, se concluye que la metodología de este trabajo puede ser usada para futuras investigaciones mejorando las fuentes de información, lo que se puede hacer generando datos continuos a partir de bases de información del Banco Mundial, Fondo Monetario Internacional, United States Patent and Trademark Office, entre otras.

\section{Referencias bibliográficas}

Arredondo, F., Vázquez, J. C., y De la Garza, J. (2016). Factores de innovación para la competitividad en la Alianza del Pacífico. Una aproximación desde el Foro Económico Mundial. Estudios Gerenciales, 32(141), 299-308. https:// doi.org/10.1016/j.estger.2016.06.003

Bengt-Åke, L. (Ed.) (1992). National systems of innovation: Towards a theory of innovation and interactive learning. Printer Publishers.

Castro-Gonzáles, S., Peña-Vinces, J. C., y Guillen, J. (2016). The competitiveness of Latin-American economies: Consolidation of the double diamond 
theory. Economic Systems, 40(3), 373-386. https://doi.org/10.1016/j. ecosys.2015.10.003

Cazallo, A. M., Barragán, C. E., Meñaca, I., Lechuga, J. I., Martínez, H. R., y Uribe, C. M. (2019). Mercosur y la Alianza del Pacífico. Dos modelos de competitividad-país. Revista Espacios, 40(18), 26-137.

Cazallo, A. M., y Salazar, E. J. R. (2018). Análisis macroeconómico de los países de la Alianza del Pacífico (20112015). Revista de Ciencias Sociales (Ve), XXIV(2), 37-55.

Cleff, T. (2019). Applied statistics and multivariate data analysis for business and economics: A modern approach using SPSS, Stata, and Excel. Springer Nature Switzerland.

Freeman, C. (1987). Technology policy and economic performance: Lesson from Japan. Pinter Publisher.

Geldes, C., Felzensztein, C., y PalaciosFenech, J. (2017). Technological and non-technological innovations, performance and propensity to innovate across industries: The case of an emerging economy. Industrial Marketing Management, 61, 55-66. https://doi.org/10.1016/j. indmarman.2016.10.010

Hair, J. F., Black, W. C., Babin, B. J., y Anderson, R. E. (2019). Multivariate Data Analysis. Cengage.

Heredia, J. A., Geldes, C., Kunc, M. H., y Flores, A. (2018). New approach to the innovation processin emerging economies: The manufacturing sector case in Chile and Peru. Technovation, 79, 35-55. https://doi.org/10.1016/j. technovation.2018.02.012

Jiménez, A., y Geldes, C. (2019). Los desafíos de la innovación en Latinoamérica. Journal of Technology Management \& Innovation, 14(4).
http://dx.doi.org/10.4067/S0718$\underline{27242019000400003}$

Lall, S. (2001). Competitiveness indices and developing countries: An economic evaluation of the global competitiveness report. World Development, 29(9), 1501-1525. https://doi.org/10.1016/S0305750X(01)00051-1

León, J. L., y Ramírez, J. J. (2014). La Alianza del Pacifico. Alcances, competitividad e implicaciones para América Latina. Friedrich-Ebert-Stiftung.

López, D. (2019). Notas críticas sobre las relaciones entre universidad, gobierno e industria. Revista de Ciencias Sociales (Ve), XXV(4), 40-50.

Montoya, O. (2004). Schumpeter, innovación y determinismo tecnológico. Scientia et Technica, X(25), 209-213.

Moon, H. C., Rugman, A. M., y Verbeke, A. (1998). A generalized double diamond approach to the global competitiveness of Korea and Singapore. International Business Review, 7(2), 135-150. https://doi.org/10.1016/S09695931(98)00002-X

Navarro-Caballero, M., Hernández-Fernández, L., Navarro-Manotas, E., y HernándezChacín, J. (2020). Innovación en las micro, pequeñas y medianas empresas familiares del sector manufacturero del Atlántico-Colombia. Revista de Ciencias Sociales (Ve), XXVI(4), 124144. $\quad$ https://doi.org/10.31876/rcs. $\underline{\mathrm{v} 26 \mathrm{i} 4.34653}$

Nelson, R. R., y Winter, S. G. (1982). An evolutionary theory of economic change. Harvard University Press.

Nikzad, R. (2013). Canadian Patent Profile: Some explorations in patent statistics. World Patent Information 35(3), 201-208. https://doi.org/10.1016/j. $\underline{\text { wpi.2013.03.001 }}$ 
Garcia Ochoa, Juan José; Ochoa Vazquez, Ivan y Valenzuela Valenzuela, Alejandro

Innovación en economías latinoamericanas: Análisis comparativo con respecto a Corea del Sur

Olavarrieta, S., y Villena, M. G. (2014). Innovation and business research in Latin American: An overview. Journal of Business Research, 67(4), 489-497. $\quad$ https://doi.org/10.1016/j. ibusres.2013.11.005

Oliver, R., y Stezano, F. (2017). Innovación como campo de estudio en Latinoamérica 2010-2017: Brasil, Chile y México. Journal of Technology Management \& Innovation, 12(4), 7079. http://dx.doi.org/10.4067/S0718$\underline{27242017000400008}$

Organización para la Cooperación y el Desarrollo Económicos - OCDE (2005). Manual de Oslo: Guía para la recogida e interpretación de datos sobre innovación. OCDE-Eurostat. http://www.itq.edu.mx/convocatorias/ manualdeoslo.pdf

Oropeza, A. (Coord.) (2011). BRICS El dificil camino entre el escepticismo y el asombro. Cámara de DiputadosUniversidad Nacional Autónoma de México,.

Parada, J. E., Ganga, F. A., y Rivera, Y. Y. (2017). Estado del arte de la innovación social: Una mirada a la perspectiva de Europa y Latinoamérica. Opción, 33(82), 563-587.

Peña-Vinces, J. D. C. (2009). Análisis comparativo de la competitividad de las economías del Perú y Chile desde un enfoque global. Journal of Economics, Finance and Administrative Science, 14(27), 87-104.

Pino, C., Felzensztein, C., Zwerg-Villegas, A. M., y Arias-Bolzmann, L. (2016). Non-tecnological innovations: Market performance of exporting firms in South America. Journal of Business Research, 69(10), 4385-4393. https:// doi.org/10.1016/j.jbusres.2016.03.061

Porter, M. E. (1990). The competitive advantage of nations. Harvard
Business Review (9), 76-97.

Porter, M. E (2008). On competition: Updated and expanded edition. Harvard Business School Publishing Corporation.

Rojas-Santoyo, F., Elizalde-Bobadilla, L., y Jiménez-García, D. (2018). Política de desarrollo productivo y fomento a la innovación de las organizaciones en el marco de los subsistemas - industria y ciencia y tecnología, para el caso de Colombia, Brasil y Corea del Sur. Revista Espacios, 39(50), 20-34.

Schumpeter, J. A. (1967). Teoría del desenvolvimiento económico. Fondo de Cultura Económica.

Vega, J. M., Fernández, I., Gutiérrez, A., y Manjarres, L. A. (25-28 de octubre de 2005). Los determinantes de la innovación tecnológica en la empresa: Una aproximación a través del concepto de capacidad de absorción. ALTEC 2005. XI Seminario Latino Iberoamericano de Gestión Tecnológica, Salvador de Bahía, Brasil.

World Economic Forum - WEF (2011). The Global Competitiveness Report 2011-2012. WEF. https://www3. weforum.org/docs/WEF GCR Report_2011-12.pdf

World Economic Forum - WEF (2012). The Global Competitiveness Report 20122013. WEF. https://www3.weforum. org/docs/WEF GlobalCompetitivenes sReport 2012-13.pdf

World Economic Forum - WEF (2013). The Global Competitiveness Report 20132014. WEF. https://www3.weforum. org/docs/WEF GlobalCompetitivenes sReport 2013-14.pdf

World Economic Forum - WEF (2014). The Global Competitiveness Report 20142015. WEF. https://www.weforum. org/reports/global-competitiveness-

Licencia de Creative Commons Atribución 4.0 Internacional (CC BY 4.0) https://creativecommons.org/licenses/by/4.0/deed.es 
report-2014-2015

World Economic Forum - WEF (2015). The Global Competitiveness Report 20152016. WEF. https://www3.weforum. org/docs/gcr/2015-2016/Global Competitiveness Report_2015-2016. pdf

World Economic Forum - WEF (2016). The Global Competitiveness Report 2016-2017. WEF. https://www3.
weforum.org/docs/GCR20162017/05FullReport/TheGlobalComp etitivenessReport2016-2017 FINAL. pdf

World Economic Forum - WEF (2017). The Global Competitiveness Report 20172018. WEF. https://www.weforum.org/ reports/the-global-competitivenessreport-2017-2018 